\title{
Chemically inhomogeneous photoionization modelling of the planetary nebula SMC N87
}

Yiannis G. Tsamis and Daniel Péquignot

LUTH, Laboratoire l'Univers et ses Théories, associé au CNRS (FRE 2462) et à l'Université Paris 7, Observatoire de Paris-Meudon, F-92195 Meudon Cédex, France

We constructed dual abundance photoionization models of SMC N87 along the principles laid out by Péquignot et al. (2002) in their modelling of the galactic PN NGC 6153. Recent spectroscopic studies have necessitated this approach if one wants to interpret correctly the faint, heavy element optical recombination lines (ORLs) seen in the spectra of $\mathrm{PNe}$ and $\mathrm{H}$ II regions. The semi-empirical analysis of Tsamis et al. $(2003,2004)$ has shown that in N87 the abundances of $\mathrm{C}$ and $\mathrm{O}$, relative to $\mathrm{H}$, derived from ORLs are a factor of 2-3 larger than those derived from collisionally excited lines (CELs). This discrepancy can be explained by introducing chemical inhomogeneities in the nebula in the form of hydrogen-deficient, cold plasma regions.

SMC N87 is an almost round nebula with no hints of significant internal structure and a very faint nucleus (cf. HST imaging by Stanghellini et al. 2003). We modelled the PN by combining 4 spherically symmetric computations with the code NEBU, using IUE and optical line fluxes from the NTT 3.5-m (Tsamis et al. 2003), AAT 3.9-m (Barlow 1987), and HST facilities (Stanghellini et al. 2003).

We consider a scenario in which the central star at some point in its evolution ejected into the nebula $\mathrm{H}$-deficient material, which originated as nucleosynthetic products brought up to the post-AGB stellar surface by the 3rd dredge up. This H-poor (metal-rich) gas was immersed in the more 'normal' abundance inner regions of the $\mathrm{PN}$, condensing into relatively dense optically thin knots. These, owing to their high metallicity, rapidly cooled down to temperatures much lower than the ambient gas, largely via the emission of infrared fine structure transitions. The model comprises: (1) a H-deficient sector (C1), with covering factor $\omega / 4 \pi=0.70$, optical depth $\tau=0.31$ at $1 \mathrm{Ryd}$, outer radius $7.14 \times 10^{16} \mathrm{~cm}$, and a small filling factor, $f$; (2) A usual-composition shell (C2), with $\omega / 4 \pi=0.87$, $\tau=5.5$, outer radius $3.07 \times 10^{17} \mathrm{~cm}$, and $f=1$ from which a region coinciding with $\mathrm{C} 1$ is removed; (3) The gas pressure is the same in $\mathrm{C} 1$ and $\mathrm{C} 2$ corresponding to conditions of isobaric cooling for $\mathrm{C} 1$; (4) The nebula is radiation bounded overall. Emission from low ionization species like [O I] and [O II] led us to include peripheral clumps in a sector with $\omega / 4 \pi=0.13$, and 'infinite' optical depth; (5) The primary spectrum is a black body $\left(L_{B B}=2.05 \times 10^{37} \mathrm{erg} \mathrm{s}^{-1}, T_{B B}=63.8 \mathrm{kK}\right)$ with a flux cut-off for frequencies above the $\mathrm{He}^{+}$limit (4 Ryd) since no He II lines are observed.

The mean electron densities and temperatures in $\mathrm{C} 1$ and $\mathrm{C} 2$ are: $\left(T_{\mathrm{e}}, N_{\mathrm{e}}\right)$ $=\left(6300 \mathrm{~K}, 33710 \mathrm{~cm}^{-3}\right)$ and $\left(12950 \mathrm{~K}, 2900 \mathrm{~cm}^{-3}\right)$, respectively. The mass of the $\mathrm{H}$-poor gas is $0.0027 \mathrm{M}_{\odot}$ vs. $0.365 \mathrm{M}_{\odot}$ in the normal component. Therefore, 
even though $\mathrm{C} 1$ has enhanced abundances of oxygen and carbon relative to $\mathrm{C} 2$ ( 6 and 21 times solar respectively, by number, relative to $\mathrm{H}$ ), the mean elemental abundances in the PN approximate those returned by the semi-empirical method for $t^{2}=0$ (cf. Table 1). The model reproduces exactly the C and O ORLs, which are severely underestimated in chemically homogeneous models.

Table 1. Elemental abundances in SMC N87 (relative to $\mathrm{H}$ )

\begin{tabular}{lccccccc}
\hline & $\mathrm{He}$ & $\mathrm{C}$ & $\mathrm{N}$ & $\mathrm{O}$ & $\mathrm{Ne}$ & $\mathrm{S}$ & $\mathrm{Ar}$ \\
\hline avg. Model & 0.089 & 8.56 & 7.14 & 8.02 & 7.24 & 6.00 & 5.39 \\
$t^{2}=0$ & 0.097 & 8.58 & 7.04 & 8.03 & 7.03 & $*$ & 5.32 \\
\hline
\end{tabular}

SMC N87 is poor in carbon, nitrogen and argon with respect to non-type I SMC PNe and has a rather large ionized mass. Only $65 \%$ of the original C was converted to $\mathrm{N}$ during the 1st dredge-up, instead of the $100 \%$ expected SMC conversion efficiency. The surface enhancement of $\mathrm{C}$ during the $3 \mathrm{rd}$ dredgeup was only $\Delta \mathrm{C} / \mathrm{H}=3.56 \times 10^{-4}$, which is atypical for SMC PNe, resembling rather that of a Galactic PN. For $\Delta \mathrm{He} / \Delta \mathrm{C}=10$ (Boothroyd \& Sackmann 1988) however, the predicted $\mathrm{He} / \mathrm{H}$ ratio is 0.087 , in agreement with our model value of 0.089 .

A potential difficulty with the scenario of a late stellar ejection as the origin of the knots is that the $\mathrm{C} / \mathrm{O}$ ratio in the $\mathrm{H}$-poor gas $(=1.8)$ is smaller than that in the normal gas $(=3.4)$, when the opposite might be expected. We also find that while the $\mathrm{He} / \mathrm{H}$ number ratio in the knots cannot be larger than $\sim 0.14$ (otherwise the He I fluxes are overestimated), it could take values as low as 0.083, indicating that $\mathrm{He}$, along with $\mathrm{H}$, might also be depleted in the knots. This opens the way for alternative explanations as to the origins of the metalrich gas, which could include a scenario involving a family of photoevaporating planetesimals in the inner regions of SMC N87.

\section{References}

1. Barlow M. J., 1987, MNRAS, 227, 161

2. Boothroyd A. I., Sackmann I.-J., 1988, ApJ, 328, 653

3. Péquignot D., Amara M., Liu X.-W., Barlow M. J., Storey P. J., Morisset C., Torres-Peimbert S., Peimbert M., 2002, RMxAA (Ser. de Conf.), 12, 142

4. Stanghellini L., Shaw R. A., Balick B., Mutchler M., Blades J. C., Villaver E., 2003, ApJ, 596, 997

5. Tsamis Y. G., Barlow M. J., Liu X.-W., Danziger I. J., Storey P. J., 2003, MNRAS, 345,186

6. Tsamis Y. G., Barlow M. J., Liu X.-W., Storey P. J., Danziger I. J., 2004, MNRAS, in press (astroph/0404280) 